\title{
Influência de agentes antioxidantes na resistência de união de substratos clareados
}

- Murilo Baena Lopes ${ }^{1}$ Klissia Romero Felizardo², Letícia Cristina Brigantini ${ }^{1}$, Sandrine Bittencourt

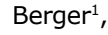
Laísa Araújo Cortines Laxe ${ }^{3}$ Luciana Andrea Salvio ${ }^{3}$

${ }^{1}$ Departamento de Odontologia Restauradora, Faculdade de Odontologia, Universidade Norte do Paraná - UNOPAR. Londrina, Paraná

${ }^{2}$ Departamento de Odontologia Restauradora, Faculdade de Odontologia, Universidade Paranaense - UNIPAR. Londrina, Paraná

${ }^{3}$ Departamento de Odontologia Restauradora, Universidade Federal de Juiz de Fora. Juiz de Fora, Minas Gerais

\section{$\triangle \quad$ Luciana Salvio}

Rua Sampaio, 346 apto 701

Centro

CEP: $36013-240$

Juiz de Fora - MG

円 luciana.salvio@ufjf.edu.br
Submetido: $30 / 11 / 2018$

Aceito: $31 / 12 / 2018$

\section{RESUMO}

A busca pela estética tem levado muitos pacientes ao consultório odontológico, sendo o clareamento de dentes vitais alternativa não invasiva, quando comparado à microabrasão, facetas e coroas, além de proporcionar elevado índice de sucesso para o tratamento de dentes escurecidos. Porém, há necessidade de espera de 14-21 dias para a realização de novas restaurações após o clareamento, uma vez que o efeito oxidante nos tecidos dentários pode gerar alterações estruturais no esmalte e redução da resistência de união. Este intervalo de tempo muitas vezes, não condiz com a expectativa dos pacientes, gerando insatisfação. Diante disso, estudos têm sido conduzidos com o objetivo de minimizar ou até mesmo anular o efeito adverso do oxigênio residual, possibilitando a execução pós-clareamento de restaurações adesivas com segurança e eficácia, reduzindo o tempo de espera. Uma das alternativas tem sido a utilização de agentes antioxidantes. Sendo assim, esse trabalho teve como objetivo revisar a literatura em relação aos agentes antioxidantes utilizados, bem como a eficácia dos mesmos na resistência adesiva de materiais restauradores ao substrato dental clareado. Esta revisão foi realizada através de buscas por artigos científicos, em bases de dados como Scielo, Pubmed/ Medline e Cochrane Library, sem limitação de data. Foram abrangidos, no estudo, trabalhos que envolvessem os temas "clareamento dental", "clareadores", "esmalte dentário", "dentina", "antioxidantes" e "resistência à tração", buscando analisar sua relevância clínica e científica. Avaliações que não se enquadravam no assunto principal e respectivos itens avaliados foram excluídas da revisão. Concluiu-se que a utilização de compostos antioxidantes, como o ascorbato de sódio $10 \%$ (gel e solução), proantocianidinas, catalase, chá verde $10 \%$ e alfa-tocoferol tem demonstrado efetividade na reversão dos valores de resistência de união ao esmalte clareado quando aplicados por 10 minutos (ascorbato em solução e proantocianidinas), 20 minutos (catalase), 60 minutos (chá verde, ascorbato gel e alfa-tocoferol), após o clareamento.

Palavras-chave: antioxidantes, clareamento dental, clareadores, resistência à tração

\section{ABSTRACT}

The search for esthetic has led many patients to the dental office, becoming the whitening of vital teeth a noninvasive alternative when compared to microabrasion, veneers and crowns, besides providing high success rate for the treatment of discolored teeth. However, there is a necessity to wait for 14-21 days to carry out new restorations after bleaching, since the oxidizing effect on dental tissues can cause structural changes in the enamel and reduction of bond strength. This time interval often does not match the expectations of patients, causing dissatisfaction. In view of this, studies have been conducted in order to minimize or even cancel the adverse effect of residual oxygen, enabling the post-bleaching performance of adhesive restorations safely and effectively, reducing the waiting time. One of the alternatives has been the use of antioxidant agents. Thus, this study aimed to review the literature in relation to the antioxidant agents used as well as their effectiveness in bond strength of restorative dental materials to the bleached substrates. This review was performed by searching for scientific articles in databases such as Scielo, PubMed / Medline and Cochrane Library, without limitation of date. It was included in the study, researches involving the themes "Tooth whitening", "Bleaching agents", "Enamel", "Dentin", "Antioxidants" and "Tensile Strength", searching to analyze its clinical and scientific relevance. The assessments that did not fit the main subject and adjacent items evaluated were excluded from the review. It can be concluded that the use of antioxidant compounds, such as $10 \%$ sodium ascorbate (gel and solution), proanthocyanidins, catalase, $10 \%$ green tea and alpha-tocopherol, have shown effectiveness in reversion of the bond strength values to bleached enamel when applied for 10 minutes (ascorbate solution and proanthocyanidins), 20 minutes (catalase), 60 minutes (green tea, gel ascorbate and alpha-tocopherol), after bleaching.

Keywords: antioxidants, tooth whitening, bleaching agents, tensile strength 


\section{INTRODUÇÃO}

Em Odontologia, o novo padrão estético é representado por dentes brancos, bem contornados e corretamente alinhados. Sendo assim, alterações dentárias que comprometam esse padrão, podem trazer sérias consequências frente a imagem social do paciente. Dependendo do significado emocional que essas alterações têm para o indivíduo, podem mudar suas relações interpessoais, causando profundas mudanças em seus padrões de autoaceitação e autoimagem, com reflexos profundos sobre sua autoestima (TIN-OO; SADDKI; HASSAN, 2011).

As pigmentações dentárias podem ser decorrentes da combinação de fatores extrínsecos e intrínsecos. Os fatores extrínsecos estão associados à permeabilidade do esmalte dental, sendo adquiridas durante a fase póseruptiva pela ingestão de substâncias de baixo peso molecular, como café, vinho tinto, chá preto, chimarrão, refrigerantes a base de cola, tabaco, acúmulo de placa e alimentos com corantes (WATTS; ADDY, 2001; JOINER, 2004).

Os fatores intrínsecos estão associados à permeabilidade da dentina, sendo gerados na fase pré-eruptiva em decorrência da excessiva ingestão de determinados medicamentos (tetraciclina e minociclina) durante a maturação do germe dental, assim como a alta ingestão de flúor (WATTS; ADDY, 2001; JOINER, 2004). Além disso, podem estar associados às condições hereditárias e congênitas como hipoplasia de esmalte, amelogênese imperfeita e dentinogênese imperfeita (VISCIO et al., 2000); substâncias usadas no tratamento de canal; traumas dentais que ocasionam hemorragia interna e pigmentação pelos componentes metálicos do amálgama (WATTS; ADDY, 2001; JOINER, 2004).

Com o tempo, os dentes sofrem um processo de escurecimento fisiológico devido à obliteração normal e gradual dos túbulos dentinários, frente à idade cronológica do paciente. Isso faz com que esses túbulos, observados mais amplos em pacientes jovens, tornemse cada vez mais obliterados, alterando a coloração dentária inicial (JOINER, 2006).

Diante de tais fatores, consolidam o clareamento de dentes vitais como sendo, muitas vezes, o tratamento de primeira escolha frente as alterações de cor em esmalte e/ou dentina (JOINER, 2006; TREDWIN; NAIK; LEWIS; SCULLY, 2006; ALQAHTANI, 2014). Este procedimento visa à utilização de compostos à base de peróxido de hidrogênio e/ ou peróxido de carbamida em diferentes apresentações, concentrações e tempos ou formas de aplicação (JOINER, 2006; ALQAHTANI, 2014).

As soluções de peróxido de carbamida são extremamente instáveis na cavidade oral e imediatamente se dissociam em peróxido de hidrogênio e uréia. O peróxido de hidrogênio, que é um forte agente oxidante, se degrada em oxigênio e água enquanto a uréia se degrada em amônia e dióxido de carbono. Os radicais livres gerados nessas reações químicas de oxidação e redução (oxigênio e hidrogênio) quebram as macromoléculas de pigmentos em moléculas cada vez menores e opticamente mais claras, sendo eliminadas por difusão na intimidade do elemento dentário (CHEN; XU; SHING, 1993).

Baseado no alto número de pessoas que apresentam restaurações estéticas em resina composta, surgiram vários estudos comprovando que pacientes que são submetidos ao tratamento clareador não poderiam trocar ou fazer novas restaurações, dentro de um período de tempo de 7 dias (BARBOSA et al., 2008; TABATABAEI et al., 2011; SALOMONE et al., 2012), 10 dias (SOUZAGABRIEL et al., 2010), 14 dias (CADENARO et al., 2006; SALOMONE et al., 2012) e 21 dias (CAN-KARABULUT; KARABULUT, 2011; GULLER; YAZDI; KOOHESTANIAN, 2012).

Este fato ocorre pela presença de radicais livres no interior dos túbulos dentinários durante e após o término do tratamento (BASTING et al., 2004; TREDWIN; NAIK; LEWIS; SCULLY, 2006; JOINER, 2007; ALQAHTANI, 2014). Esse efeito oxidativo gera alterações microestruturais como porosidades e depressões, além de leve queda na dureza superficial (TREDWIN; NAIK; LEWIS; SCULLY, 2006; JOINER, 2007; ALQAHTANI, 2014), proporcionando baixa resistência de união (DOMINGUEZ, 2012), devido à redução da infiltração do sistema adesivo, causando assim uma diminuição do número, tamanho e da qualidade dos prolongamentos resinosos. Uma vez que esses radicais livres inibem a polimerização do compósito (ATTIN, 2004; PEGORARO et al., 2011), podem gerar microinfiltrações (MORTAZAVI, 2011).

Essa discrepância entre a cor das restaurações antigas e a cor do dente clareado, proporciona um grande incômodo ao paciente que procura o tratamento restaurador logo após o clareamento. Para diminuir este tempo de espera, estudos vêm sendo realizados com o propósito de possibilitar esta troca imediata de restaurações com a utilização de substâncias que acelerem a liberação do oxigênio remanescente (GAMA et al., 2006).

Uma das alternativas tem sido o uso de substâncias antioxidantes como a catalase (GOPINATH et al., 2013), o ascorbato de sódio (TORRES; KOGA; BORGES, 2006; TURKUN et al., 2009; KIMYAI et al., 2010) e ácido ascórbico (NOMOTO et al., 2006; MURAGUCHI et al., 2007), o extrato de sementes de uva (proantocianidina) (VIDHYA et al., 2011), o chá verde (BERGER et al., 2013), o resveratrol (VENTURINI et al., 2010), a-tocoferol (vitamina E) (SASAKI et al., 2009), entre outros.

O uso de antioxidantes vem sendo objeto de pesquisas odontológicas como uma alternativa para amenizar o efeito negativo do uso de peróxidos na resistência de união dos compósitos resinosos ao substrato dental 
clareado. Desta forma, o presente trabalho objetivou revisar os principais agentes antioxidantes empregados em odontologia, bem como a eficácia dos mesmos no que concerne a resistência adesiva.

\section{REVISÃO DE LITERATURA}

A técnica de clareamento mais utilizada em dentes vitalizados com alterações de cor intrínseca ou extrínseca, até 1989 , consistia no condicionamento do esmalte com ácido fosfórico $37 \%$ seguido do tratamento com o peróxido de hidrogênio $30 \%\left(\mathrm{H}_{2} \mathrm{O}_{2}\right)$, associado ou não à aplicação de uma fonte de calor (HAYWOOD; HEYMANN, 1989).

Entretanto, essa técnica apresentava inconvenientes como a necessidade de várias sessões em consultório, a realização de um cuidadoso isolamento absoluto para proteção dos tecidos moles e o polimento do esmalte após o tratamento, dadas as alterações causadas pelo condicionamento ácido e pelos subprodutos do peróxido de hidrogênio. Além dos inconvenientes apresentados pela técnica, a aplicação de calor em dentes vitalizados gera grandes preocupações em relação à vitalidade pulpar do elemento dentário (MCEVOY, 1989).

Devido aos efeitos adversos e desvantagens fornecidas por essa técnica, diferentes produtos e sistemas de tratamento foram introduzidos. Em 1989, a técnica caseira com moldeiras pré-formadas foi introduzida e idealizada por Haywood \& Heymann. Utilizava-se como principal agente clareador o peróxido de carbamida $10 \%$, associado ao carbopol, na forma de gel. Esse gel funciona como uma fonte de peróxido de hidrogênio de baixa concentração, mas por um período prolongado, permitindo assim uma ação lenta e contínua, com pouca chance de sensibilidade dentária para o paciente (FRANCI et al., 2010).

O clareamento caseiro (de auto-aplicação) é administrado pelo paciente com orientações do dentista, sendo prescritas concentrações de peróxido de carbamida, que variam de $10 \%$ a $22 \%$ (ROBERTO et al., 2011). Essa técnica é cada vez mais comum, uma vez que o seu sucesso está ligado ao fato de ser uma alternativa de fácil aplicação, segura, conservadora, econômica e eficaz (SIQUEIRA; REZENDE; CALIXTO; KOSSATZ, 2011). Entretanto, apresenta desvantagens, como o uso da moldeira, o comprometimento do paciente para uma boa evolução do tratamento e o tempo da aplicação maior quando comparado com o clareamento de consultório, onde é aplicada uma concentração mais alta do produto por menos tempo (SIQUEIRA; REZENDE; CALIXTO; KOSSATZ, 2011; BRISO et al., 2014).

Apresenta em seu composto glicerol ou propilenoglicol (que irá atuar no transporte e responde a $85 \%$ do produto), agente aromático, ácido fosfórico ou cítrico e Carbopol. A fundamental importância do Carbopol é tornar a substância mais espessa, aumentando a adesão do gel à estrutura dental (SOARES et al., 2008). A substância em que o Carbopol está presente libera oxigênio mais lentamente, sendo recomendado o uso noturno. Uma liberação mais lenta faz com que o produto aja por mais tempo, tornando-se mais eficaz (SOARES et al., 2008). Quando as substâncias não possuem o Carbopol, o oxigênio é liberado com maior velocidade.

Com a intenção de tornar mais rápido o procedimento de clareamento de dentes vitais, surgiu a técnica de consultório ("in-office", em inglês), chamada de clareamento assistido. É realizada exclusivamente no consultório do cirurgião-dentista, onde são utilizadas concentrações mais altas, de peróxido de hidrogênio variando entre $30 \%$ a $38 \%$ ou de peróxido de carbamida com concentrações variando entre $35 \%$ a $37 \%$ (FRANCI et al., 2010).

Dentre as vantagens relacionadas ao clareamento de consultório destaca-se uma melhor administração do tratamento, pois não depende da colaboração do paciente, bem como maior controle da aplicação em locais de retração gengival, locais estes propícios a reações de hipersensibilidade (PERDIGÃO; BARATIERI; ARCARI, 2004; SOARES et al. 2008). Como pontos negativos, o clareamento de consultório geralmente necessita de mais de uma visita ao consultório para melhores resultados, tornando-o um procedimento de custo elevado (KUGEL; KASTALI, 2000). Além disso, há maior índice de recidiva de cor a curto prazo (DAWSON et al., 2011).

Essa técnica foi associada a fontes de luz com o objetivo de "acelerar" o procedimento. O que a maior parte da literatura científica tem mostrado em trabalhos laboratoriais (GOMES et al., 2009) e em clínicos (in vivo) (MARSON et al., 2008; ALOMARI; EL DARAA, 2010) é que o uso dessas fontes de luz é desnecessário para o procedimento, e que o "acelerar" que elas podem proporcionar é desprezível, não justificando o investimento nesse tipo de equipamento (FRANCI et al., 2010; ALMEIDA et al., 2012; HAHN et al., 2013).

Segundo Buchalla; Attin (2007), a aceleração do processo clareador pode ocorrer pela fotólise, que é a excitação direta da molécula de peróxido de hidrogênio pela luz, causando uma maior liberação de radicais hidroxila; ou pela termocatálise, que causa a aceleração da liberação de radicais livres por meio do calor. No entanto, a energia requerida para que essas reações ocorram torna seu uso na cavidade oral difícil e inseguro, devido à grande possibilidade de causar danos pulpares.

Uma tendência observada é o uso do próprio peróxido de hidrogênio para o clareamento de auto-aplicação com moldeiras, variando de 6 a 9,5\%, por um tempo menor que o observado para o peróxido de carbamida, não excedendo 1 hora, e peróxido de hidrogênio para uso em consultório em concentrações entre 15 e 25\%, apenas variando o tempo de aplicação, ou seja, mantendo o 
mesmo produto clareador por um período único e longo de 30 a 50 minutos, contrastando com a técnica original em que se preconiza utilizar normalmente três trocas de gel em intervalos de até 15 minutos (FRANCI et al., 2010).

Essa possibilidade de aplicação por um período único e longo está relacionada ao maior controle de $\mathrm{pH}$ durante o tempo de contato do gel clareador com as estruturas dentais. $\mathrm{O} \mathrm{pH}$ se mantém por volta de 7 ou mais alcalinizado, o que permite a geração de radicais livres de peróxido de hidrogênio mais eficientes em remover pigmentos (PRICE; SEDAROUS; HILTZ, 2000).

Para promover uma maior estabilidade de cor, tem sido preconizada a associação das duas técnicas (caseira e de consultório). Essa associação (conhecida em inglês como "jump start") se inicia com uma sessão de clareamento em consultório, realizada com peróxido de hidrogênio em altas concentrações e, posteriormente, o paciente conclui o tratamento com a técnica caseira (DELIPERI; BARDWELL; PAPATHANASIOU, 2004). A associação das duas técnicas para o clareamento de dentes vitais possibilita obter melhores resultados, pois reduz o tempo de tratamento e diminui a irritação gengival e a sensibilidade dental (DELIPERI; BARDWELL; PAPATHANASIOU, 2004).

A teoria do clareamento envolve a dissociação do agente clareador com a liberação de radicais livres de oxigênio que penetram através das porosidades dos primas de esmalte e dos túbulos dentinários, provavelmente devido à permeabilidade dental e ao baixo peso molecular (cerca de $30 \mathrm{~g} / \mathrm{mol}$ ) (GARCIA et al., 2012). Através de uma reação de oxidação, os pigmentos, que são macromoléculas de alto peso molecular, são fragmentados em cadeias moleculares menores, com isso através de um processo de difusão são parcialmente eliminados da estrutura dental. Além disso, essas moléculas absorvem menos luz, tornando o dente mais claro (GOPINATH et al., 2013).

Estudos tem demonstrado que alguns resíduos químicos ativos, dentre eles o oxigênio dos peróxidos de agentes clareadores podem permanecer aprisionados na estrutura dental e a eliminação apenas com água, que não possui ação antagonista ao oxidante, tem se mostrado ineficaz para a eliminação desse peróxido de hidrogênio residual (TABATABAEI et al., 2011; GARCIA et al., 2012), proporcionando redução na resistência adesiva de materiais resinosos nos casos que exigem a substituição de restaurações logo após o clareamento (DISHMAN et al., 1994; SPYRIDES et al., 2000; CAVALLI et al., 2001; BASTING et al., 2004; CAVALLI et al., 2004; MIGUEL et al., 2004).

De acordo com Torres et al. (2006) o oxigênio residual pode diminuir a resistência à fratura do esmalte e interferir na formação da camada híbrida, pois o oxigênio poderia ficar preso dentro desta durante a polimerização. Além disso, também podem ocorrer outras alterações, tais como, mudança na textura de superfície, na composição, porosidade e dureza do esmalte (TABATABAEI et al., 2011).

Para avaliar a resistência de união ao esmalte dental, ensaios mecânicos têm sido utilizados e sua confiabilidade tem sido demonstrada em diversos estudos (OZCAN; VALANDRO, 2012; VANDERLEI; BOTTINO; VALANDRO, 2013). Um destes ensaios mecânicos sugeridos é a microtração, que foi introduzida na odontologia na década de 90 , no entanto algumas pesquisas tem demonstrado que esse teste não deve ser utilizado no esmalte dental, devido à fragilidade inerente do tecido, podendo gerar resultados diferentes do real, pois podem provocar trincas no esmalte (SHIMADA; KIKUSHIMA; TAGAMI, 2002). Outro método que tem sido preconizado para analisar pequenas áreas de interface adesiva em esmalte é o ensaio de microcisalhamento (MCDONOUGH et al., 2002), que utiliza uma força de cisalhamento aplicada através de uma fina alça de aço inoxidável.

Para que a adesão não seja comprometida, estudos tem indicado ser necessário um período de espera entre o término do tratamento clareador e a realização de restaurações adesivas para que o oxigênio residual possa ser eliminado, sendo que este período varia de acordo com diferentes autores, de 7 dias (TABATABAEI et al., 2011), 10 dias (SOUZA-GABRIEL et al., 2011), 14 dias (CADENARO et al., 2006; SALOMONE et al., 2012) e 21 dias (CAN-KARABULUT; KARABULUT, 2011; GULLER; YAZDI; KOOHESTANIAN, 2012).

Entretanto, Este intervalo de tempo não condiz com a expectativa do paciente em relação à conclusão do tratamento estético. Uma alternativa para a redução deste período de espera é o uso de agentes antioxidantes, que agem como estabilizadores de radicais livres (BRAZ et al., 2011), permitindo que a adesão do compósito ao dente clareado seja realizada com sucesso imediatamente após o tratamento clareador, pois neutralizam o fator etiológico responsável pela incompleta polimerização dos monômeros resinosos (TURKUN et al., 2009).

$\mathrm{Na}$ tentativa de aumentar a resistência de união ao dente clareado e diminuir o tempo de espera para a realização do tratamento, vários tipos de antioxidantes foram estudados, dentre eles a catalase (GOPINATH et al., 2013), o ascorbato de sódio (TORRES; KOGA; BORGES, 2006; TURKUN et al., 2009; KIMYAI et al., 2010) e ácido ascórbico (NOMOTO et al., 2006), o extrato de sementes de uva (proantocianidina) (VIDHYA et al., 2011), o chá verde (BERGER et al., 2013), o resveratrol (VENTURINI et al., 2010), a-tocoferol (vitamina E) (SASAKI et al., 2009), entre outros.

A neutralização dos radicais livres é um processo natural que ocorre no organismo e resulta na oxidação do agente neutralizador, que é classificada em: prevenção em tempo integral e a desintoxicação (ativa e passiva). O papel da prevenção de tempo integral é de 
evitar o excesso de produção de radicais livres e se dá pela inativação de moléculas, que são responsáveis pela sua geração. Já o de desintoxicação é baseado em três enzimas que compõem o sistema de base de um sistema de defesa antioxidante, que são a superóxido dismutase, catalase e peroxidase, que podem ser encontradas em células em que o estresse oxidativo é maior, como citosol, mitocôndrias e peroxissomas (SCRIVER et al., 1989).

A catalase é uma enzima que naturalmente existe no corpo humano para regularizar os níveis de peróxidos, pode ser encontrada no fígado, rim e eritrócitos. Esta enzima decompõe rapidamente o peróxido de hidrogênio em oxigênio e água (TORRES et al., 2006).

Rotstein (1993), foi o precursor do uso de antioxidantes, na tentativa de eliminar os efeitos adversos do oxigênio residual pós-clareamento. Ele analisou o efeito do uso da enzima catalase aplicada por 3 minutos após o clareamento dental interno, comparando-a com o efeito de apenas sucessivas lavagens com água, e concluiu que nos dentes tratados com catalase houve uma significativa redução do peróxido de hidrogênio residual.

O ascorbato de sódio é um hidro-antioxidante, encontrado em fluídos biológicos, derivado do ácido ascórbico. É composto de vitamina $\mathrm{C}$ e sódio, sendo que seus sais são considerados não-tóxicos e amplamente utilizados na indústria alimentar como antioxidantes (LAI et al., 2001, LAI et al., 2002; KAYA; TURKUN, 2003). Em medicina cosmética é usado como indutor da formação de colágeno e na odontologia, como agente co-preventivo de doença periodontal.

Tem-se conhecimento de que o ascorbato de sódio é uma substância instável, de cor amarelada e que se oxida rapidamente, podendo levar a possíveis manchamentos nos dentes recém-clareados (DSM, 2001). No intuito de evitar esse possível efeito adverso, se propõe a utilização de um gel antioxidante à base de ascorbato de sódio estabilizado (PI0502546-0), de cor brancotranslúcida, quimicamente conhecido como ascorbato de trisódio monofosfatado ou ascorbil fosfato. O ascorbil fosfato é um pó branco, facilmente solúvel em água em concentração de até $50 \%$ em pH básico (DSM, 2002).

Agem como estabilizadores de radicais livres, promovendo a redução de reatividade das espécies derivadas do oxigênio e nitrogênio e, assim, é capaz de prevenir o dano oxidativo para importantes macromoléculas biológicas, tais como o DNA, proteínas e lípidos (SOHEILI et al., 2003).

Em relação ao período de aplicação do antioxidante, estudos (TURKUN; KAYA, 2004; BULUT; KAYA; TURKUN, 2005) encontraram que a resistência de união da resina composta aumentou após o tratamento de dentes clareados com ascorbato de sódio a $10 \%$ por 10 minutos. Outros estudos (KIMYAI; VAFIZADEH, 2006; PAUL; ROSALINE; BALAGOREL, 2007) demonstraram que o uso de ascorbato de sódio gel ou solução a $10 \%$ por 3 horas neutralizou o efeito oxidativo do clareamento, aumentando a resistência de união do esmalte. Entretanto, o uso de ascorbato de sódio por esse período somado ao tempo utilizado para o clareamento pode tornar-se clinicamente inaceitável (LAI et al., 2002).

Esse tempo de aplicação foi discutido por Kaya et al. (2008), que apontaram que a eficiência da remoção dos radicais livres pelo ascorbato de sódio a $10 \%$ acontece a partir de 60 minutos de aplicação direta sobre a estrutura clareada. Dabas et al. (2011), relataram que o aumento da resistência de união a dentes clareados é diretamente proporcional ao tempo de aplicação do ascorbato de sódio.

Existem duas apresentações de ascorbato de sódio, o hidrogel de ascorbato de sódio e a solução de ascorbato de sódio. A melhora dos valores de resistência de união foram observados em ambas as formas utilizando a mesma concentração no clareamento de dentes vitais (CAN-KARABULUT; KARABULUT, 2011).

O ácido ascórbico apresentou-se capaz de elevar a resistência de união em esmalte dental clareado com peróxido de carbamida quando aplicado por 180min. Mas não apresentou eficácia quando utilizado sobre dentes que sofreram clareamento com peróxido de hidrogênio (NOMOTO et al., 2006). Os resultados de Muraguchi et al. (2007) foram divergentes e o ácido ascórbico foi eficiente para o uso após o clareamento em dentes vitais com peróxido de hidrogênio ou perborato de sódio quando associado a um sistema adesivo autocondicionante de 2 passos.

Outro antioxidante pesquisado na busca de diminuir o tempo entre o clareamento dental e a restauração em resina composta, assegurando uma eficiente resistência de união é a Proantocianidina (complexos oligoméricos de proantocianidinas - OPCs) derivados do óleo de semente de uva. A proantocianidina (PA) tem sido reportada por estabilizar e aumentar a ligação cruzada exógena de tecidos a base de colágeno (CASTELLAN et al., 2011), pelas ligações de hidrogênio entre o hidroxilo fenólico e os grupos cabonilo e amida da proteína (HAN et al., 2003).

Este composto ocorre naturalmente nos metabólitos de plantas, sendo amplamente disponível em frutas, vegetais, castanhas, sementes, flores e cascas (FINE, 2000). Apesar da PA ser conhecida primeiramente por sua atividade antioxidante, este composto tem sido relatado também por demonstrar ações antibacteriana, antiviral, anticarcinogênica, anti-inflamatória, antialérgica e vasodilatadora (FINE, 2000).

Além disso, o extrato da semente de uva rico em PA aumentou as propriedades mecânicas da dentina desmineralizada, tais como: módulo de elasticidade (CASTELLAN et al., 2010a; dos SANTOS et al., 2011a; dos SANTOS et al., 2011b), nanodureza (dos SANTOS et al., 2011a; dos SANTOS et al., 2011b), resistência coesiva 
(CASTELLAN et al., 2010a; LIU et al., 2011) e resistência de união dente restauração (CASTELLAN et al., 2010a; FANG et al., 2012; SRINIVASULU et al., 2012).

A aplicação de proantocianidinas à $5 \%$ em substratos que foram clareados com peróxido de hidrogênio a $38 \%$ por $10 \mathrm{~min}$ apresentou resultados de resistência de união significantemente maiores em comparação ao uso do ascorbato de sódio a 10\% (VIDHYA et al., 2011).

O chá verde, originário da planta Camellia Sinensis, já evidenciou resultados promissores na redução da erosão dentária (MAGALHÃES et al., 2009), como agente antimicrobiano (YOO et al., 2011), na redução da inflamação gengival (MARUYAMA et al., 2011) e atualmente vem sendo estudado quanto ao seu poder antioxidante para aumentar a resistência de união de dentes clareados (BERGER, et al., 2013). O mesmo contém principalmente flavonóides ou catequinas de epigalocatequina galato (EGCG), epigalocatequina (EGC), epicatequina galato (ECG) e epicatequina (EC) (CHAN et al., 2011; HORŽIU et al., 2009). Estudos relatam que o chá verde apresenta propriedades antioxidantes, e estas são atribuídas às catequinas das EGCG e EGC (HORŽIU et al., 2009).

Essa capacidade antioxidante de compostos fenólicos é devido principalmente às suas propriedades de óxido redução podendo assim absorver e neutralizar radicais livres, quelando o oxigênio singlete (molécula de oxigênio em estado altamente energético, que pode ser gerada pela transferência de energia de uma molécula excitada por luz visível ou ultravioleta) e triplete ou decompondo peróxidos aumentando assim a resistência de união do composto ao esmalte clareado (DEGÁSPARI; WASZCZYNSKYJ, 2004).

O resveratrol (3,4,5-trihidroxiestibeno) é uma fitoalexina produzida por plantas, sua produção ocorre em resposta ao stress causado pelo ataque fúngico, dano mecânico ou irradiação de luz ultravioleta (WANG; $\mathrm{XU}$; LI, 2008). Esse bioativo é sintetizado por plantas sob duas formas isômeras: trans-resveratrol e cisresveratrol; para fins terapêuticos é obtido de extratos de uvas francesas Vitis vinifera, bem como em bebidas oriundas da planta, pois estas apresentam altas concentrações deste composto antioxidante (JANG; CAI, 1997), sendo superior aos tradicionais suplementos como ácido ascórbico e vitamina E (WANG; XU; LI, 2008; VENTURINI et al., 2010; KOO; MONTMINY, 2006).

Seu mecanismo de ação envolve o sequestro de espécies reativas de oxigênio (EROS), atuando como quelante de metais e modulando enzimas envolvidas em estresse oxidativo (BLANDER; GUARENTE, 2004). A atividade antioxidante de compostos fenólicos, como o resveratrol, é principalmente devida às suas propriedades de óxido-redução, as quais podem desempenhar um importante papel na absorção e neutralização de radicais livres, quelando o oxigênio ou decompondo peróxidos (BRENNA; PAGLIARINI, 2001;
ZHENG; WANG, 2001).

A vitamina C (VC) e a vitamina $E(V E)$, hidrossolúvel e lipossolúvel, respectivamente, são consideradas antioxidantes efetivos. Estas vitaminas estão envolvidas na manutenção de reações anti-oxidativas e na proteção contra a peroxidação lipídica causada por espécies reativas de oxigênio (EROS) formados durante a resposta inflamatória ou mesmo gerados durante as atividades metabólicas normais das células (UPADHYAY; MISRA, 2009).

A VE é composta por uma mistura de Tocoferóis e Tocotrienóis, sendo que ambos apresentam quatro isômeros (a, $\beta, \square$ e $\delta$ ) (AGGARWAL et al., 2010). Porém, a ação antioxidante é mediada pelo isômero alfa-Tocoferol (a-T), o qual possui a capacidade de incorporar-se na membrana celular e impedir a propagação da peroxidação lipídica (UPADHYAY; MISRA, 2009). Alguns pesquisadores avaliaram o efeito protetor do a-T e verificaram sua capacidade de estabilizar a membrana celular, e assim impedir a propagação do estresse oxidativo, aumentando a viabilidade celular e a quantidade de antioxidantes endógenos (AZZI; STOCKER, 2000; NEGIS et al., 2007; UPADHYAY; MISRA, 2009).

Diversos estudos sobre a ação do a-T têm sido desenvolvidos dentro da área médica devida sua ação coadjuvante no tratamento de algumas doenças, tais como aterosclerose, diabetes, mal de Alzheimer e câncer, pois impede, ou pelo menos reduz eventos oxidativos (MUNTEANU; RICCIARELLI; ZINGG, 2004; MAZLAN et al., 2006; SHINGU et al., 2011).

Com relação ao clareamento dental, a incorporação de a-T na membrana celular poderia prevenir 0 desencadeamento da peroxidação lipídica provocada pelos EROS que penetram através do esmalte e dentina e atingem o tecido pulpar (BUETTNER, 1993). Estudos comprovaram que a incorporação de a-T na membrana de células (Linfócito T) ocorre entre 24 e 27 horas (SAITO et al., 2003), sendo que para as células vermelhas esta incorporação já se apresenta completa no período de 24 horas (CHEESEMAN et al., 1995).

Majd et al. (2003) avaliaram o efeito protetor de alguns antioxidantes, como o Trolox, análogo da vitamina $E$, especificamente do isômero a-Tocoferol, e do ascorbato de sódio contra os efeitos tóxicos de materiais odontológicos de origem resinosa. Para isto, células de linhagem fibroblástica oriunda de gengiva humana foram utilizadas para o cultivo celular. Os extratos obtidos dos materiais resinosos após 24 horas foram colocados em contato com as células e mantidos por mais 24 horas para posterior análise da viabilidade celular. Além disso, os autores quantificaram a presença de íons metálicos e de antioxidantes endógenos como a Glutationa. Observou-se que o ascorbato de sódio não apresentou efeito protetor em relação aos materiais que continham íons metálicos na sua composição. Porém, o 
a-T hidrossolúvel na concentração de $3 \mathrm{mM}$ apresentouse eficaz contra os efeitos tóxicos de materiais resinosos.

A utilização de substâncias antioxidantes após a técnica de clareamento dental pode ser importante, não só para restaurar resistência de união após o tratamento, mas para a possível proteção de células pulpares contra a agressão imposta pelos componentes tóxicos do clareamento realizado com materiais com capacidade de difusão transdentinária (SASAKI; FLORIO; BASTING, 2009).

\section{DISCUSSÃO}

O clareamento dental é um dos procedimentos clínicos mais procurados pelos pacientes, visto que a questão estética ocupa lugar de destaque nos dias atuais. Suas técnicas de aplicação já estão consolidadas pela literatura e o acesso ao procedimento clareador, quer seja realizado em consultório ou de forma caseira com uso de moldeiras, é cada vez mais fácil. Por ser uma técnica simples, de fácil manuseio e com uso de agentes clareadores de baixa concentração, o clareamento caseiro ainda é o mais utilizado (HAYWOOD; HEYMANN, 1989; SOARES, 2008).

No entanto, muitas das vezes o clareamento dental por si só não é capaz de assegurar a expectativa dos pacientes, levando à associação do mesmo com técnicas restauradoras em resinas compostas. Porém, grande dificuldade está relacionada à baixa adesividade dos materiais restauradores estéticos ao esmalte recentemente clareado, o que faz necessário tempo de espera entre a conclusão do clareamento e o procedimento restaurador subsequente.

A perda de adesividade da resina ao esmalte está relacionada com a possível presença de oxigênio residual, que interfere na polimerização da mesma (LAI et al., 2002; CAVALLI, 2001), formando bolhas geradoras de tensão (TORNECK et al., 1992; DISHMAN et al., 1994; LAI et al., 2002) e uma menor adaptação e penetração dos tags em esmalte (PERDIGÃo et al., 1998). Essas alterações que ocorrem na estrutura do esmalte são resultado também de um aumento da porosidade que se manifesta por uma aparência sobre-condicionada com perda da forma prismática (BEM-AMAR et al., 1995). Além disso, outros fatores importantes que poderiam ter contribuido para a diminuição da resistência de união que inclui perda de cálcio, diminuição da microdureza e alterações na substância orgânica (TIMPAWAT et al., 2005).

Com o objetivo de evitar falhas adesivas na interface material restaurador e superfície dental, um período de espera variável de até três semanas é recomendado (TITLEY; TORNECK; RUSE, 1992; CAVALLI et al., 2001; ATTIN et al., 2004; UNLU; COBANKARA; OZER, 2008) uma vez que a redução na resistência de união da resina composta ao esmalte ou dentina após o tratamento clareador tem sido demonstrada como temporária (MCGUCKIN; THRMOND; OSOVITZ, 1992).
Uma variedade de antioxidantes tem sido pesquisada por se mostrar capaz de remover o peróxido de oxigênio residual, de modo a neutralizar os radicais livres remanescentes (DROGE, 2002). Uma substância antioxidante pode ser definida como sendo uma substância química que inibe o processo de oxidação, ou qualquer substância que, quando presente em baixa concentração, comparada à do substrato oxidável, diminui ou inibe significativamente a oxidação daquele substrato. Do ponto de vista biológico, pode-se definir antioxidantes como compostos que protegem sistemas biológicos contra os efeitos potencialmente danosos de processos ou reações que promovem a oxidação de macromoléculas de estruturas celulares (ABDALLA, 2000).

Dentre os antioxidantes mais citados encontram-se o ácido ascórbico e seu derivado, o ascorbato de sódio. Contudo o ácido ascórbico não tem sido recomendado devido a sua acidez (BRISO et al., 2012).

Resultados apresentados por Nomoto et al. (2006) sugerem que o uso do ácido ascórbico melhora a adesão quando aplicado durante $2 \mathrm{~h}$, mas os resultados de resistência à microtração apresentaram-se inferiores aos grupos sem tratamento, e quando realizado durante 3 horas em antioxidante não apresentou diferenças significativas para o grupo sem clareamento. Esses resultados vão de encontro com os resultados de Lai et al. (2002) que também obtiveram bons resultados com um tempo mínimo de aplicação do agente antioxidante (durante 3 horas).

Em contrapartida, a solução de ascorbato de sódio a $10 \%$ tem demonstrado ser efetiva para neutralizar o oxigênio singleto e os subprodutos gerados na superfície da dentina, favorecendo a adesão e aumento da resistência de união do sistema adesivo à dentina, além de evitar falhas adesivas e compensar a diminuição da resistência à fratura em dentes endodonticamente tratados e clareado (LAI et al., 2001; KIMYAI; VALIZADEH, 2006; KIMYAI; VALIZADEH, 2008; TURKUN; CELIK; KAYA; ARICI, 2009; KHOROUSHI; FEIZ; KHODAMORADI, 2010).

Tabatabaei et al. (2011), ao avaliarem o uso do ascorbato de sódio a $10 \%$ como antioxidante aplicado por 5 e 10 minutos após o clareamento dental com peróxido de carbamida a $35 \%$ em incisivos bovinos e posterior restauração adesiva, comparado com a imersão em água destilada por 7 dias e restauração após esse tempo, obtiveram como resultado de cisalhamento, que os grupos com o uso do agente antioxidante apresentaram menor resistência de união que o grupo que foi mantido em água destilada por 7 dias.

Por outro lado, no estudo de Freire et al. (2009) foi verificado que a reação entre peróxido de hidrogênio e ascorbato de sódio foi rápida e que 5 minutos foi tempo suficiente para que o antioxidante fizesse a redução dos efeitos adversos do gel clareador. Os autores relatam que um tempo de aplicação de 5 minutos é adequado para condições clínicas e também permite que procedimentos de adesão sejam iniciados mais cedo, eliminando o tempo 
de espera de 2-3 semanas após o clareamento sugerido por muitos autores (CAVALLI et al., 2001; BASTING et al., 2004; MIGUEL et al., 2004).

No que diz respeito ao tempo de aplicação desta substância antioxidante (AS) alguns autores (TURKUN; KAYA, 2004; BULUT et al., 2006; GOKÇEB et al., 2008) propuseram o uso de ascorbato de sódio durante 10 minutos e relataram que os efeitos adversos do agente clareador foram invertidos com êxito. Por outro lado, alguns autores relatam que este período independe do tipo e da concentração do peróxido utilizado, sendo o seu uso preconizado até $1 / 3$ do tempo de aplicação do gel de clareamento (LAI et al., 2002; TURKUN et al., 2004).

O estudo realizado por Khoroushi; Aghelinejad (2011) detectou a reversão dos efeitos adversos dos radicais livres com o uso do ascorbato a $10 \%$ associado com sistemas auto condicionantes ou não, em três situações, imediato, uma semana, e com o uso do ascorbato. Independente do sistema adesivo utilizado quando associados com o ascorbato de sódio mesmo de forma imediata apresentaram resultados satisfatórios para o teste de microcisalhamento.

Miranda et al. (2013) ao avaliarem o efeito da aplicação do antioxidante ascorbato de sódio por 60 minutos após o clareamento dental, verificaram que a resistência de união foi igual estatisticamente ao de dentes não clareados e ao de dentes clareados e imersos em saliva humana por 7 dias. No entanto, esse tempo de 60 minutos, torna essa estratégia inviável para o uso na prática clínica.

Arantes et al. (2005) utilizaram a catalase após o clareamento dental e testaram a resistência adesiva desses espécimes obtendo resultados promissores, pois o grupo com catalase apresentou os mesmos valores de resistência de união que o grupo controle, que não foi clareado. Torres et al. (2006), também utilizando a catalase e outros agentes antioxidantes como o ascorbato de sódio, bicarbonato de sódio e acetona compararam a resistência adesiva entre esmalte e resina composta e concluíram que a aplicação da catalase por 20 minutos foi o agente antioxidante que proporcionou a maior resistência de união, corroborando com os estudos de Gopinath et al. (2013).

Garcia et al. (2012), testaram a atividade antioxidante de diversos agentes como: ascorbato de sódio, ácido ascórbico, bicarbonato de sódio, catalase (Neutralize, FGM- Produtos Odontológicos, Joinvile, SC, Brasil), entre outros. Obtiveram como resultado que todos esses agentes citados, apresentaram atividade antioxidante significativa.

Torres; Koga; Borges (2006), avaliaram o efeito neutralizador de seis agentes antioxidantes, por 20 minutos (ascorbato de sódio 10\% -AS; Catalase-CA; Glutationa Peroxidase-GP; Acetona-AC; Etanol-ET; Bicarbonato de sódio 7\%- BS) sobre a resistência de união do esmalte clareado com o gel de peróxido de hidrogênio 35\%. Foi observado que apenas a aplicação da Catalase (CA) resultou em aumento significativo da resistência de união.

O aumento da resistência de união dente-restauração após o pré-tratamento da dentina desmineralizada com extratos ricos em proantocianidinas (complexos oligoméricos de proantocianidinas - OPCs) derivados do óleo de semente de uva, tem sido reportado, pela indução de ligações cruzadas no colágeno (AL-AMMAR et al., 2009; CASTELLAN et al., 2010). A interação entre proantocianidinas e proteínas tem sido postulada por quatro mecanismos: (1) interações covalentes, (2) interaçõe iônicas, (3) interações por pontes de hidrogênio, ou (4) interações hidrófobas (BEDRANRUSSO et al., 2007). A aplicação de proantocianidinas $5 \%$ em substratos que foram clareados com peróxido de hidrogênio a $38 \%$ por 10 minutos apresentou resultados de resistência de união significativamente maiores em comparação ao uso do ascorbato de sódio a 10\% (VIDHYA et al., 2011). Resultados semelhantes foram encontrados quando o OPCs foi associado a sistemas adesivos (ABRAHAM et al., 2013).

Ozelin et al. (2013), avaliaram a capacidade de dois agentes antioxidantes (chá verde $10 \%$ e ascorbato de sódio a $10 \%$ na forma de gel, sendo os mesmos utilizados por 15 minutos, 30 minutos e 1 hora) para inverter os valores da resistência de união após clareamento com peróxido de carbamida $10 \%$. De acordo com os resultados obtidos apenas o tempo de aplicação de 1 hora foi efetivo no aumento da resistência de união de acordo com o trabalho de Carreira et al. (2012). O tempo de aplicação de 15 e 30 minutos do gel de ascorbato de sódio $10 \%$ e do gel de chá verde $10 \%$ respectivamente, não diferiram estatisticamente dos valores de resistência de união ao esmalte do grupo só clareado com peróxido de carbamida a $10 \%$. Tais resultados corroboram com os achados de Kaya et al. (2008); Schwertner et al. (2016) e Berger et al. (2013) que avaliaram o efeito de diferentes tempos de aplicação do ascorbato de sódio a $10 \%$ (na forma de gel e solução) e chá verde a $10 \%$, sendo que somente a partir do tempo de 60 minutos foi efetivo para a reversão dos valores de resistência de união ao esmalte clareado.

Cavalli et al. (2005) verificou que o chá verde na forma de gel foi capaz de remover peróxido residual, que interfere na adesão de resina ao esmalte após clareamento e inibe a polimerização de resina, permitindo que o procedimento adesivo seja executado imediatamente após o clareamento.

Upadhyay; Misra (2009), avaliaram o efeito do alfatocoferol ( $\mathrm{a}-\mathrm{T}$ ) em contato com células odontoblastóides MDPC-23 na utilização do peróxido de hidrogênio em diferentes tempos de pré-tratamento. Observou-se que o a-T quando em contato com células MDPC-23 por 1 hora, foi capaz de reduzir o efeito tóxico do peróxido de hidrogênio. Tais dados corroboram com os estudos de Lima et al. (2010), os quais avaliaram a vitamina $\mathrm{C}$, quando em contato com células odontoblastoídes MDPC-23 por 1 hora, na utilização do peróxido de carbamida.

Vargas et al. (2013), avaliaram quais concentrações de a-T $(1,3,5$ e $10 \mathrm{mM})$ associadas a variados tempos de pré-tratamento $(1,4,8$ e 24 horas) de células MDPC-23, podem prevenir, ou pelo menos reduzir, os efeitos tóxicos do peróxido de hidrogênio $(\mathrm{PH})$ sobre este tipo celular. Os 
resultados mostraram efeito protetor do a-T para todos os tempos de pré-tratamento e em todas as concentrações testadas. De maneira geral, observou-se, para o grupo onde as células foram expostas apenas ao $\mathrm{PH}$, há persistência de $41 \%$ do metabolismo celular. Por outro lado, foi observado, que quando as células MDPC-23 foram submetidas ao prétratamento com diferentes concentrações de a-T e em seguida expostas ao $\mathrm{PH}$, houve importante redução dos efeitos tóxicos do produto sobre as células em cultura. Este dado confirmou a capacidade do a-T em proteger as células odontoblastóides MDPC-23 contra os efeitos oxidantes do $\mathrm{PH}$. Além disso, foi demonstrado que quanto maior o tempo de contato das células com o $\mathrm{a}-\mathrm{T}$, menores são as concentrações necessárias para obter-se o efeito protetor.

Dessa forma, o potencial terapêutico da VE é amplo, sendo que a proposta de administrar esse composto vai além da sua ação antioxidante, capaz de inibir a peroxidação lipídica, pois também pode atuar como agente antiinflamatório na polpa, minimizando outros efeitos colaterais negativos que podem ser causados pelo clareamento dental (VARGAS et al., 2013).

\section{CONCLUSÃO}

A presente revisão permite concluir que os compostos antioxidantes empregados nas pesquisas odontológicas analisadas neste trabalho, sugerem que o uso da solução de ácido ascórbico influencia positivamente na resistência de união ao substrato dental clareado, quando o mesmo é aplicado por 2 a $3 \mathrm{~h}$, porém não tem sido recomendado em decorrência da sua acidez e longo período de aplicação. Em contrapartida, a solução de ascorbato de sódio a $10 \%$ tem demonstrado efetividade quando aplicado por 5 e 10 minutos após o clareamento. Tais dados são similares no caso da utilização das proantocianidinas (OPCs) em uma concentração de $5 \%$ pelo tempo de 10 minutos. Quanto à catalase a mesma apresentou efeito antioxidante e efetividade de adesão quando aplicada por 20 minutos. Outros estudos mostraram que tanto o chá verde a $10 \%$, como o ascorbato de sódio a $10 \%$ (na forma de gel e solução) somente apresentam efeito para a reversão dos valores de resistência de união ao esmalte clareado, a partir do tempo de 60 minutos, estando em similaridade com o antioxidante alfa-tocoferol (a-T) ao se utilizar maiores concentrações, por outro lado, quanto maior o tempo de contato das células com o a-T (24h), menores são as concentrações necessárias para obter-se o efeito protetor.

\section{REFERÊNCIAS}

ABRAHAM, S. et al. Effect of grape seed extracts on bond strength of bleached enamel using fifth and seventh generation bonding agents. Journal of International Oral Health, v. 5, n. 6, p.101-107, dez. 2013.
AGGARWAL, B.B. et al. Tocotrienols, the vitamin E of the 21 st century: its potencial against cancer and other chronic diseases. Biochemical Pharmacology, v. 80, n. 11, p. 1613-1631, dez. 2010.

AL-AMMAR, A.; DRUMMOND, J.L.; BEDRAN-RUSSO, A.K. The use of collagen cross-linking agents to enhance dentin bond strength. Journal of Biomedical Material part B, v. 91, n. 1, p. 419-424, out. 2009.

ALMEIDA, L.C.A.G. et al. Ocurrence of sensivity during at-home and in-office tooth bleaching therapies with or without use of light sources. Acta Odontológica Latinoamericana, v. 25, n. 1, p. 3-8, abr. 2012.

ALOMARI, Q.; EL DARAA, E. A randomized clinical trial of in-office dental bleaching with or without light activation. The Journal of Contemporary Dental Practice, v. 11, n. 1, p. 17-24, jan. 2010.

ALQAHTANI, M.Q. Tooth-bleaching procedures and their controversial effects: A literature review. Saudi Dent Journal, v.26, n.2, p. 33-46, abr. 2014.

ARANTES, A.C. et al. Efeito da Catalase sobre a Força de União adesiva de dentes bovinos expostos ao peróxido de carbamida 10\%. Revista de Clínica e Pesquisa Odontológica, v.2, n.1, p. 47-49, jul./set. 2005.

ATTIN, T. et al. Effect of bleaching on restorative materials and restorations - a sytematic review. Dental Materials, v.20, n.9, p. 852-861, nov. 2004.

AZZI, A.; STOCKER, A. Vitamin E: non-antioxidant roles. Progress in Lipid Research, v. 39, n. 3, p. 231-55, mai. 2000.

BARBOSA, C.M. et al. Influence of time on bond strength after bleaching with $35 \%$ hydrogen peroxide. The Journal of Contemporary Dental Practice, n.9, n.2, p.81-88, fev. 2008.

BASTING, R.T. et al. Shear bond strength of enamel treated with seven carbamide peroxide bleaching agents. Journal of Esthetic and Restorative Dentistry, v. 16, n. 4, p. 250-259, jul. 2004.

BEDRAN-RUSSO, A.K. et al. Application of crosslinkers to dentin collagen enhances the ultimate tensile strength. Journal of Biomedical Material part B, v. 80, n.1, p. 268-272, jan. 2007.

BEN-AMAR, A. et al. Effect of mouthguard bleaching on enamel surface. American Journal of Dentistry, v. 8, n. 1, p. 29-32, fev. 1995.

BERGER, S. B. et al. Can green tea be used compromised bond strength after bleaching? European Journal of Oral Sciences, v. 121, n. 4, p. 377-81, ago. 2013.

BLANDER, G.; GUARENTE, L. The Sir2 family of protein deacetylases. Annual Review of Biochemistry, v.73, n.1, p. 417-435, jul. 2004. 
$B R A Z$, R. et al. Influence of antioxidants on stress of bonding agents in recently whitened teeth. Acta Odontológica Latinoamericana, v. 24, n. 3, p. 252-257, dez. 2011.

BRENNA, O.V.; PAGLIARINI, E. Multivariate analyses of antioxidant power and polyphenolic composition in red wines. Journal of Agricultural and Food Chemistry., v. 49, n.10, p. 4841-4844, out. 2001.

BRISO, A.L.F. et al. Análise do clareamento dental caseiro realizado com diferentes produtos: relato de caso. Revista Odontológica de Araçatuba., v. 35, n. 1, p. 49-54, jan/jul. 2014.

BRISO, A. L. F. et al. Effect of sodium ascorbate on tag formation in bleached enamel. The Journal of Adhesive Dentistry, v. 14, n.1, p.19-23, fev. 2012.

BUCHALLA, W.; ATTIN, T. External bleaching therapy with activation by heat, light or laser-a systematic review. Dental Materials, v.23, n.5, p.586-596, mai. 2007.

BUETTNER, G.R. The pecking order of free radicals and antioxdants: lipid peroxidation, a-tocopherol and ascorbate. Archives of Biochemistry and Biophysics, v. 300, n. 2, p. 535-543, fev. 1993.

BULUT, H.; KAYA, A.D.; TURKUN, M. Tensile bond strength of brackets after anti-oxidant treatment on bleached teeth. European Journal of Orthodontics, v. 27, n. 5, p. 466-471, out. 2005.

BULUT, H.; TURKUN, M.; KAYA, A.D. Effect of an antioxidizing agent on the shear bond strength of brackets bonded to bleached human enamel. American Journal of Orthodontics \& Dentofacial Orthopedics, v. 129, n. 2, p. 266-72, fev. 2006.

CADENARO, M. et al. Influence of whitening on the degree of conversion of dental adhesives on dentin. European Journal of Oral Sciences, v.114, n.3, p. 257-262, jun. 2006.

CAN-KARABULUT, D.C.; KARABULUT, B. Influence of activated bleaching on various adhesive restorative systems. Journal of Esthetic and Restorative Dentistry, v. 23, n. 6, p. 399-408, dez. 2011.

CARREIRA, R.P.S. et al. Efeito do chá verde na resistência de união do esmalte submetido ao tratamento clareador caseiro. 2012. 39f. Dissertação (Mestre em Dentística Preventiva e Restauradora) - Universidade Norte do Paraná, Londrina, 2012.

CASTELLAN, C.S. et al. Long-term stability of dentin matrix following treatment with various natural collagen cross-linkers. Journal of the Mechanical Behavior of Biomedical Materials, v. 4, n. 7, p. 1343-1350, out. 2011.

CASTELLAN, C.S. et al. Mechanical characterization of proanthocyanidin-dentin matrix interaction. Dental Materials, v. 26, n.10, p. 968-973, out. 2010.
CAVALLI, V. et al. The effect of elapsed time following bleaching on enamel bond strength of resin composite. Operative Dentistry, v. 26, n. 6, p. 597-602, nov./dez. 2001.

CAVALLI, V.; DE CARVALHO, R.M.; GIANNINI, M. Influence of carbamide peroxide based bleaching agents on the bond strength of resin enamel/dentin interfaces. Brazilian Oral Research, v. 19, n. 1, p. 23-29, jan./mar. 2005.

CAVALLI, V.; GIANNINI, M.; CARVALHO, R. M. Effect of carbamide peroxide bleaching agents on tensile strength of human enamel. Dental Materials, v.20, n.8, p.733-739, out. 2004.

CHAN, E.W. et al. Antioxidant and antibacterial properties of green, black, and herbal teas of Camellia sinensis. Pharmacognosy Research, v.3, n.4, p. 266-272, out./dez. 2011.

CHEESEMAN, K.H. et al. Biokinetics in humans of RRR-alphatocopherol: the free phenol, acetate ester, and succinate ester forms of vitamin E. Free Radical Biology and Medicine, v. 19, n. 5, p. 591-8, nov. 1995.

CHEN, J.H.; XU, J.W.; SHING, C.X. Decomposition rate of hydrogen peroxide bleaching agents under various chemical and physical conditions. The Journal of Prosthetic Dentistry, v. 69, n. 1, p. 46-48, jan. 1993.

DABAS, D.; PATIL, A.C.; UPPIN, V.M. Evaluation of the effect of concentration and duration of application of sodium ascorbate hydrogel on the bond strength of composite resin to bleached enamel. Journal of Conservative Dentistry, v. 14, n. 4, p. 356-360, out. 2011.

DAWSON, P.F. et al. A clinical study comparing the efficacy and sensitivity of home vs combined whitening. Operative Dentistry, v. 36, n. 5, p. 460-466, set./out. 2011.

DELIPERI, S.; BARDWELL, D.N.; PAPATHANASIOU, A. Clinical evaluation of a combined in-office and take-home bleaching system. The Journal of American Dental Association, v.135, n.5, p. 628-634, mai. 2004.

DISHMAN, M. V.; COVEY, D. A.; BAUGHAN, L. W. The effects of peroxide bleaching on composite to enamel bond strength. Dental Materials, v. 10, n. 1, p.33-36, jan. 1994.

DOMINGUEZ, J.A. et al. Ultrastructural evaluation of enamel after dental bleaching associated with fluoride. Microscopy Research and Technique, v.75, n.8, p. 1093-1098, ago. 2012.

DOS SANTOS, P.H.; KAROL, S.; BEDRAN-RUSSO, A.K. Longterm nano-mechanical properties of biomodified dentin-resin interface components. Journal of Biomechanics., v. 44, n.9, p.1691-1694, jun. 2011a. 
DOS SANTOS, P.H.; KAROL, S.; BEDRAN-RUSSO, A.K. Nanomechanical properties of biochemically modified dentin bonded interfaces. Journal of Oral Rehabilitation., v. 38, n.7, p. 541-546, jul. 2011b.

FANG. M. et al. Biomodification to dentin by a natural crosslinker improved the resin-dentin bonds. Journal of Dentistry, v. 40, n. 6, p. 458-466, jun. 2012.

FINE, A. M. Oligomeric proanthocyanidin complexes: history, structure, and phytopharmaceutical applications. Alternative Medicine Review: A Journal of Clinical Therapeutic., v. 5, n. 2, p. 144-115, abr. 2000.

FRANCI, C. et al. Clareamento dental- Técnicas e Conceitos atuais. Revista da Associação Paulista de Cirurgiões Dentistas, $v$ especial, n. 1, p. 78-89, sem mês. 2010.

FREIRE, A. et al. Reaction kinetics of sodium ascorbate and dental bleaching gel. Journal of Dentistry, v. 37, n.12, p. 932-936, dez. 2009.

GAMA, A.M.S. et al. Restaurações adesivas em dentes pósclareados: efeito do ascorbato de sódio na remoção do $\mathrm{O} 2$. Revista Odonto Ciência, v. 21 , n. 53, p. 238-44, jul./set. 2006.

GARCIA, E. J. et al. Antioxidant activity by DPPH assay of potencial solutions to be applied on bleaching teeth. Brazilian Dental Journal, v. 23, n. 1, p. 22-27, jan./fev. 2012.

GOKÇE, B. et al. Effect of antioxidant treatment on bond strength of a luting resin to bleached enamel. Journal of Dentistry, v. 36, n.10, p. 780-785, out. 2008.

GOMES, M.N.; FRANCCI, C.; MEDEIROS, I.S. et al. Effect of light irradiation on tooth whitening: enamel microhardness and color change. Journal of Esthetic and Restorative Dentistry, v.21, n. 6, p. 387-94, dez. 2009.

GOPINATH, S. et al. Effect of bleaching with two different concentrations of hydrogen peroxide containing sweet potato extract as an additive on human enamel: An in vitro spectrophotometric and scanning electron microscopy analysis. Journal of Conservative Dentistry, v. 16, n. 1, p. 45-49, jan. 2013.

NIAT, A.B.; YAZDI, F.M.; KOOHESTANIAN, N. Effects of drying agents on bond strength of etch-and-rinse adhesive systems to enamel immediately after bleaching. The Journal of Adhesive Dentistry, v. 14, n. 6, p. 511-516, dez. 2012.

HAHN, P. et al. Efficacy of tooth bleaching with and without light activation and its effect on the pulp temperature: an in vitro study. Odontology, v. 101, n. 1, p. 67-74, jan. 2013.
HAN, B. et al. Proanthocyanidin: a natural crosslinking reagent for stabilizing collagen matrices. Journal of Biomedical Material part A, v. 65, n. 1, p. 118-124, abr. 2003.

HAYWOOD, V.B.; HEYMANN, H.O. Nightguard vital bleaching. Quintessence International, v.20, n.3, p. 173-176, mar. 1989.

HORŽIU, D. et al. The composition of polyphenols and methylxanthines in teas and herbal infusions. Food Chemistry, v.115, n.2, p. 441-448, jul. 2009.

JANG, M. et al. Cancer chemopreventive activity of resveratrol, a natural product derived from grapes. Science, v.275, n.5297, p. 218-220, jan. 1997.

JOINER, A. Review of the effects of peroxide on enamel and dentine properties. Journal of Dentistry, v.35, n.12, p. 889-896, dez. 2007.

JOINER, A. The bleaching of teeth: a review of the literature. Journal of Dentistry, v. 34, n. 7, p. 412-419, ago. 2006.

JOINER, A. Tooth colour: a review of the literature. Journal of Dentistry, v. 32, n. suplemento, p.3-12, sem mês. 2004.

KAYA, A.D.; TÜRKÜN, M.; ARICI, M. Reversal of compromised bonding in bleached enamel using antioxidant gel. Operative Dentistry, v. 33, n. 4, p. 441-447, jul./ago. 2008.

KHOROUSHI, M.; AGHELINEJAD, S. Effect of postbleaching application of an antioxidant on enamel bond strength of three different adhesives. Medicina Oral, Patología Oral y Cirugía Bucal. v. 16, n. 7, p. 990-996, nov. 2011.

KHOROUSHI, M.; FEIZ, A.; KHODAMORADI, R. Fracture resistance of endodontically-treated teeth: effect of combination bleaching and an antioxidant. Operative Dentistry, v. 35, n. 5, p. 530-537, set./out. 2010.

KIMYAI, S. et al. Comparison of the effect of hydrogel and solution forms of sodium ascorbate on orthodontic bracket-enamel shear bond strength immediately after bleaching: an in vitro study Indian Journal of Dental Research, v. 21, n. 1, p. 54-8, jan./mar. 2010.

KIMYAI, S.; VAFIZADEH. The effect of hydrogel and solution of sodium ascorbate onbond strength in bleached enamel. Operative Dentistry, v. 31, n. 4, p. 496-499, jul./ago. 2006.

KOO, S.H.; MONTMINY, M. In vino veritas: a tale of two shirts? Cell., v.127, n.6, p. 1091-1093, dez. 2006.

KUGEL, G.; KASTALI, S. Tooth-whitening efficacy and safety: a randomized and controlled clinical trial. Compendium of Continuing Education in Dentistry, v.29, n. especial, p. S16-21, quiz S42, sem mês. 2000. 
LAI, S.C. et al. Reversal of compromised bonding to oxidized etched dentin. Journal of Dental Research, v. 80, n. 10, p. 1919-1924, out. 2001.

LAI, S. C. N. et al. Reversal of compromised bonding in bleached enamel. Journal of Dental Research, v. 81, n. 7, p. 477-481, jul. 2002.

LIMA, A.F. et al. Protective effect of sodium ascorbate on MDPC23 odontoblast-like cells exposed to a bleaching agent. European Journal of Dentistry, v. 4, n. 3, p. 238-244, jul. 2010.

LIU. R. et al. The effect of transient proanthocyanidins preconditioning on the cross-linking and mechanical properties of demineralized dentin. Journal of Materials Science. Materials in Medicine, v. 22, n. 11, p. 2403-2411, nov. 2011.

MAGALHÃES, A.C. et al. Chlorhexidine and green tea extract reduce dentin erosion and abrasion in situ. Journal of Dentistry, v. 37, n. 12, p. 994-998, dez. 2009.

MAJD, E.S.; GOLDBERG, M.; STANISLAWSKI, L. In vitro effects of ascorbate and Trolox on the biocompatibility of dental restorative materials. Biomaterials, v. 24, n. 1, p. 3-9, jan. 2003.

MARSON, F.C. et al. Clinical e valuation of in-office dental bleaching treatments with and without the use of light-activation sources. Operative Dentistry, v.33, n.1, p. 15- 22, jan./fev. 2008.

MARUYAMA, T. et al. Supplementation of Green tea catechins in dentifrices suppresses gingival oxidative stress and periodontal inflammation. Archives of Oral Biology, v. 56, n. 1, p. 48-53, jan. 2011.

MAZLAN, M. et al. Comparative effects of alpha-tocopherol and gamma-tocotrienol against hydrogen peroxide induced apoptosis on primary-cultured astrocytes. Journal of the Neurological Sciences., v. 243, n.1-2, p. 5-12, abr. 2006.

MCDONOUGH, W.G. et al. A microshear test to measure bond strengths of dentin-polymer interfaces. Biomaterials, v.23, n.17, p.3603-3608, set. 2002.

MCEVOY, S.A. Chemical agents for removing intrinsic stains from vital teeth. II. Current techniques and their clinical application. Quintessence International, v. 20, n. 6, p. 379-384, jun. 1989.

MCGUCKIN, R. S; THRMOND, B. A.; OSOVITZ, S. Enamel shear Bond strengths after vital bleaching. American Journal of Dentistry, $v$. 5, n. 4, p. 216-22, ago. 1992.

MIGUEL, L. C. et al. In situ effect of $10 \%$ carbamide peroxide on resin-dentin bond strengths: a novel pilot study. Journal of Esthetic and Restorative Dentistry, v. 16, n. 4, p. 235- 241, jul. 2004.
MIRANDA, T.A.M. et al. Influence of exposure time to saliva and antioxidant treatment on bond strength to enamel after tooth bleaching: an in situ study. Journal of Applied Oral Science, v. 21, n. 6, p. 567-574, nov./dez. 2013.

MORTAZAVI, V.; FATHI, M.; SOLTANI, F. Effect of postoperative bleaching on microleakage of etch-and-rinse and self-etch adhesives. Dental Research Journal, v. 8, n. 1, p. 16-21, inverno. 2011.

MUNTEANU, A.; RICCIARELLI, R.; ZINGG, J.M. HIV protease inhibitors-induced atherosclerosis: prevention by alpha-tocopherol. International Union of Biochemistry and Molecular Biology life Life, v. 56, n. 10, p. 629-631, out. 2004.

MURAGUCHI, K. et al. Improvement of bonding to bleached bovine tooth surfaces by ascorbic acid treatment. Dental Materials, v. 26, n. 6, p. 875-881, nov. 2007.

NEGIS, Y. et al. Molecular mechanism of alphatocopherylphosphate transport across the cell membrane. Biochemical and Biophysical Research Communications, v. 359, n. 2, p. 348-353, jul. 2007.

NOMOTO, S. et al. Influence of ascorbic acid on bonding of peroxide-affecteddentin and 4-META/MMA-TBB resin. Clinical Oral Investigations, v.10, n.4, p. 325-330, dez. 2006.

OZCAN, M.; VALANDRO, L.F. Bond strength of two resin cements to titanium after diferente surface conditioning methods. General Dentistry, v. 60, n. 1, p. 6-12, jan./fev. 2012.

OZELIN, A. A. et al. Effects of Green Tea Application Time on Bond Strength after Enamel Bleaching. Brazilian Dental Journal, v. 25, n. 5, p. 399-403, set./out. 2014.

PAUL, P.; ROSALINE, H.; BALAGOPEL, S. The effect of hydrogel and solution of sodium ascorbate on the bond strength of bleached enamel. Journal of Conservative Dentistry, v. 10, n. 2, p. 4347, abr./jun. 2007.

PEGORARO, C.A.C.C. et al. Influência dos agentes clareadores na resistência adesiva de restaurações com compósitos aos tecidos dentários: momento atual. Revista Dentística Online, v. 10, n. 20, p. 11-18, sem mês. 2011.

PERDIGÃO, J.; BARATIERI, L.N.; ARCARI, G.M. Contemporary trends and techniques intooth whitening: a review. Practical Procedures \& Aesthetic Dentistry, v. 16, n. 3, p. 185-92, abr. 2004.

PERDIGÃO, J. et al. Ultra-morphological study of the interaction of dental adhesives with carbamide peroxide-bleached enamel. American Journal of Dentistry, v. 11, n. 6, p. 291-301, dec. 1998. 
PRICE, R.B.; SEDAROUS, M.; HILTZ, G.S. The pH of toothwhitening products. Journal of the Canadian Dental Association, v.66, n.8, p. 421-426, set. 2000.

ROBERTO, A.R. et al. Evaluation of tooth color after bleaching with and without light-activation. Revista Odonto Ciência, $v$. 26, n.3, p. 247-252, jul./set. 2011.

ROTSTEIN, I. Role of catalase in the elimination of residual hydrogen peroxide following tooth bleaching. Journal of Endodontics, v.19, n.11, p.567-569, nov. 1993.

SAITO, Y. et al. Cell death caused by selenium deficiency and protective effect of antioxidants. The Journal of biological chemistry, v.278, n.41, p. 39428-39434, out. 2003.

SALOMONE, P. et al. Residual oxygen releasing time from dental structure after carbamide peroxide exposure: study of the effects of a neutralizer gel. General Dentistry, n.60, p.147-50, abr. 2012

SASAKI, R. T.; FLÓRIO, F. M.; BASTING, R. T. Effect of $10 \%$ sodium ascorbate and $10 \%$ alpha-tocopherol in different formulations on the shear bond strength of enamel and dentin submitted to a home-use bleaching treatment. Operative Dentistry, v.34, n.6, p.746-752, nov./dez. 2009.

SCHWERTNER, R.C.A. et al. The effect of green tea on the shear strength of brackets after home whitening treatment. Applied Adhesion Science, v.4, p.12, sem mês. 2016.

SCRIVER, C.R. et al. The metabolic basis of inherited disease. 6th ed. New York: McGraw-Hill, p. 155-61, 1989.

SHIMADA, Y.; KIKUSHIMA, D.; TAGAMI, J. Micro-Shear bond strength of resin bonding systems to cervical enamel. American Journal of Dentistry, v.15, n.6, p.373-7, dez. 2002.

SHINGU, C. et al. EPCK1, a vitamin C and E analogue, reduces endotoxin-induced systemic inflammation in mice. The Journal of Surgical Research, v.171, n.2, p. 719-725, dez. 2011.

SIQUEIRA, M.R., et al. Associando o clareamento de consultório com o caseiro para aumentar a longevidade: relato de caso. Full Dentistry in Science, v. 2, n.7, p. 305-12, sem mês. 2011.

SOARES, F. F. et al. Clareamento em dentes vitais: uma revisão bibliográfica. Revista Saúde.com, v. 4, n.1, p.72-84, jan./jun. 2008.

SOHEILI, M. E.; GOLDBERG, M.; STANISSLAWSKI, L. In vitro effect of ascorbate and Trolox on biocompatibility of dental restorative materials. Biomaterials, v.24, n.1, p.3-9, jan. 2003.
SOUZA-GABRIEL, A.E. et al. Effect of bleaching protocols with $38 \%$ hydrogen peroxide and post-bleaching times on dentin bond strength. Brazilian Dental Journal, v.22, n.4, p.317-21, jul./ago. 2010.

SPYRIDES, G. M. et al. Effect of whitening agents on dentin bonding. Journal of Esthetic and Restorative Dentistry, v.12, n.5, p.264-270, set. 2000 .

SRINIVASULU, S. et al. Shear bond strength of composite to deep dentin after treatment with two different collagen cross-linking agents at varying time intervals. Operative Dentistry, v. $37, n$. 5, p. 485-491, set./out. 2012.

TABATABAEI, M.H. et al. Antioxidant effect on the shear bond strengh of composite to bleached bovine dentin. Brazilian Journal of Oral Sciences, v.10, n.1, p.33-36, jan./mar. 2011.

TIMPAWAT, S., et al. Effect of bleaching agents on bonding to pulp chamber dentine. International Endodic Journal, v. 38, n.4, p.211-217, abr. 2005.

TIN-OO, M.M.; SADDKI, N.; HASSAN, N. Factors influencing patient satisfaction with dental appearance and treatments they desire to improve aesthetics. BioMed Central Oral Health, v.11, p.6, fev. 2011.

TITLEY, K. C.; TORNECK, C. D.; RUSE, N. D. The effect of carbamide-peroxide gel on the shear bond strength of a microfil resin to bovine enamel. Journal of Dental Research, v.71, n.1, p.20-24, jan. 1992.

TORNECK, C. D. et al. The influence of time of hydrogen peroxide exposure on the adhesion of composite resin to bleached bovine enamel. Journal of Endodontics, v. 16, n. 3, p. 123-127, mar. 1990.

TORRES, C. R. G.; KOGA, A. F.; BORGES, A. B. The effects of antioxidant agents as neutralizers of bleaching agents on enamel bond strength. Brazilian Journal of Oral Sciences, v.5, n.16, p.971-976, jan./mar 2006.

TREDWIN, C.J. et al. Hydrogen peroxide tooth- whitening (bleaching) products: Review of adverse effects and safety issues. British Dental Journal, v. 200, n. 7, p. 371-376, abr. 2006.

TURKUN, M. et al. Can the hydrogel form of sodium ascorbate be used to reverse compromised bond strength after bleaching? The Journal of Adhesive Dentistry, v. 11, n. 1, p. 35-40, fev. 2009.

TURKUN, M.; KAYA, A.D. Effect of $10 \%$ sodium ascorbate on the shear bond strength of composite resin to bleached bovine enamel. Journal of Oral Rehabilitation, v. 31, n.12, p. 11841191, dez. 2004. 
UNLU, N.; COBANKARA, F. K.; OZER, F. Effect of elapsed time following bleaching on the shear bond strength of composite resin to enamel. Journal of Biomedical Material part B, v. 84, n. 2, p. 363-8, fev. 2008.

UPADHYAY, J., MISRA, K. Towards the interaction mechanism of tocopherols and tocotrienols (vitamin $\mathrm{E}$ ) with selected metabolizing enzymes. Bioinformation., v. 3, n. 8, p. 326-31, abr. 2009.

VANDERLEI, A.; BOTTINO, M.A.; VALANDRO, L.F. Evaluation of Resin Bond Strength to Yttriastabilized Tetragonal Zirconia and Framework Marginal Fit: Comparison of Different Surface Conditionings. Operative Dentistry, v. 39, n.1, p.50-53, jan./fev. 2013.

VARGAS, F.S. Efeito protetor da vitamina E (a-Tocoferol) contra a atividade citotóxica do peróxido de hidrogênio. 2013. 79f. Dissertação (Mestrado em Odontologia) - Universidade Estadual Paulista, Faculdade de Odontologia, 2013.

VENTURINI, C. D. et al. Resveratrol and red wine function as antioxidants in the nervous system without cellular proliferative effects during experimental diabetes. Oxidative Medicine and Cellular Longevity, v. 6, n. 3, p. 434-441, nov./dez. 2010.

VIDHYA, S. et al. Effect of grape seed extract on the bond strength of bleached enamel. Operative Dentistry, v. 36, n. 4, p. 433-438, jul./ago. 2011.

VISCIO, D. et al. Present and future technologies of tooth whitening. Compendium of Continuing Education in Dentistry, v.28, n. suplemento, p. S36-S43, sem mês. 2000.

WANG, A.; XU, Y.; LI, W. Tissue distribution and excretion of resveratrol in rat after oral administration of Polygonum cuspidatum extract (PCE). Phytomedicine, v.15, n.10, p. 859-866, out. 2008.

WATTS, A.; ADDY, M. Tooth discolouration and staining: a review of the literature. British Dental Journal, v. 190, n. 6, p. 309-16, mar. 2001.

YOO, S.; MURATA, R. M.; DUARTE, S. Antimicrobial traits of teaand cranberryderived polyphenols against Streptococcus mutans. Caries Research, v. 45, n. 4, p.327-335, set. 2011.

ZHENG, W.; WANG, S.Y. Antioxidant activity and phenolic compounds in selected herbs. Journal of Agricultural and Food Chemistry, v. 49, n.11, p. 5165-5170, nov. 2001. 